\title{
Role of Biometrics in Healthcare Privacy and Security Management System
}

\author{
Dr. Mogli Goverdhan Das \\ eHealth Management, HEARTCOM INC. Ohio, USA. \\ Ph.D., FHRIM (UK), FAHIMA (USA) Sr. Consultant eHealth Management HEARTCOM INC. (USA) \\ E-mail address: gdmogli@yahoo.com \\ $\underline{\text { www.drmogliit.com }}$
}

eHealth Sri Lanka 2010,1(suppl.1):S41

DOI: http://dx.doi.org/10.4038/sljbmi.v1i0.3694

Only the Abstract is available

\begin{abstract}
Introduction: The transformation of healthcare system to provide swift, safe, improved quality and cost contained care is experiencing multifarious problems such as passwords are meant to protect computer network systems from unauthorised use, but they also may provide a false sense of security. Why Biometric? Biometric identification systems employ used biological data, in the form of voice, face and signature scans, the most common physiological biometric include finger-scan, retina scan, hand-scan, and iris-scan. In USA, due to HIPAA requirement that physicians and healthcare professionals who use EHR are tracked every time a patient's record is accessed. Generally, you can digitally authenticate a user with any of these authentication methods: what you know, what you have, or what you are. "Who we are" refers to what is unique about us, our physical bodies as human beings. Things like: fingerprints, retinal design, facial shape, vocal wave, hand geometry, and in the future, DNA. Authentication is referred to as one-to-one matching.

Bioterrorism and Public Health: Biometrics is very helpful in two important ways. Firstly, the biometricidentification of terrorists can be distributed electronically to all potential risk areas within and outside the nation for preventive purposes. Secondly, it can help in detecting the disease, if effective, would substantially reduce the mortality, morbidity. The new trend in public health is driven by the need for early warning system, detection of surreptitious biological attacks.

Conclusion: The biometric plays a very important role in not only in maintaining privacy and security of healthcare management system but is of tremendous value in bioterrorism and public health activities in notifying infectious diseases and controlling mortality and morbidity rates by linking with curative hospital.
\end{abstract}

Keywords - Healthcare Privacy and Security Management System, EHR, HIPAA, bioterrorism, curative hospital 